\section{Intersections}

Canadian Journal of Music

Revue canadienne de musique
Intersections CANADIAN JOURAL OF MUSIC
REVUE CANADIENEE DE MUSIOUH

\title{
Le Prisonnier de Luigi Dallapiccola : derrière le politique, le religieux
}

\section{Charlotte Ginot-Slacik}

Volume 31, numéro 2, 2011

URI : https://id.erudit.org/iderudit/1013214ar

DOI : https://doi.org/10.7202/1013214ar

Aller au sommaire du numéro

\section{Éditeur(s)}

Canadian University Music Society / Société de musique des universités canadiennes

\section{ISSN}

1911-0146 (imprimé)

1918-512X (numérique)

Découvrir la revue

Citer cet article

Ginot-Slacik, C. (2011). Le Prisonnier de Luigi Dallapiccola : derrière le politique, le religieux. Intersections, 31(2), 65-78.

https://doi.org/10.7202/1013214ar
Résumé de l'article

Composé pendant la seconde guerre mondiale, Le Prisonnier de Luigi Dallapiccola constitue un tournant pour la musique italienne : premier opéra sériel italien en un temps de réaction esthétique et charge contre le fascisme, il est un modèle pour des compositeurs engagés tels que Luigi Nono et Bruno Maderna. Par-delà sa charge politique, Le Prisonnier est également l'expression d'une crise religieuse et d'un doute existentiel. L'Inquisition, institution religieuse par excellence est aussi celle qui torture le croyant au nom de la foi. Le retournement diabolique fait par le Grand Inquisiteur des principales thématiques chrétiennes vise à détruire le libre arbitre de l'autre. La manipulation du prisonnier, Christ contemporain, démontre la vacuité, voire la dangerosité de l'espérance, au nom de laquelle a été abjuré le libre arbitre. Dans l'affrontement entre le geôlier et son prisonnier s'esquisse enfin une sombre définition de la liberté car la parole de celui qui enferme n'est pas plus libre que celle de celui qui est enfermé. La mère seule, unique personnage clairvoyant de l'opéra a pu conserver sa liberté, mais à quel prix : celui de ne pas entrer dans l'action. Le doute qui émerge du Prisonnier est aussi celui de Luigi Dallapiccola, en individu directement touché par la guerre et les lois raciales, et en compositeur humaniste. L'objet de ce texte sera d'étudier la façon dont le musicien décline les thématiques religieuses mais pour les retourner totalement. Car Le Prisonnier, immense oeuvre politique, est aussi celle de l'errance et du questionnement religieux.
All Rights Reserved @ Canadian University Music Society / Société de musique des universités canadiennes, 2012
Ce document est protégé par la loi sur le droit d'auteur. L’utilisation des services d'Érudit (y compris la reproduction) est assujettie à sa politique d'utilisation que vous pouvez consulter en ligne.

https://apropos.erudit.org/fr/usagers/politique-dutilisation/ 


\title{
LE PRISONNIER DE LUIGI DALLAPICCOLA: DERRIÊRE LE POLITIQUE, LE RELIGIEUX
}

\author{
Charlotte Ginot-Slacik
}

\section{INTRODUCTION}

"Mon admiration sans borne pour Luigi Dallapiccola ; une admiration qui regarde aussi les aspects moraux et humains de ce personnage face auquel je me retrouve comme devant les grands maîtres de la Renaissance. (...) C'est de lui, des Chants de Prison, et du Prisonnier que me vient mon grand amour pour les hérétiques et les persécutés.» Ces mots de Luigi Nono illustrent la stature morale prise après la guerre par Luigi Dallapiccola, compositeur de Vol de Nuit et du Prisonnier.

Dans l'Italie des années 50, le musicien florentin s'impose comme l'archétype du compositeur chrétien, son Prisonnier devient un emblème des musiciens inspirés par la Resistenza européenne. Figure exemplaire pour Luigi Nono et Bruno Maderna, Dallapiccola incarne celui qui a emprunté la voie prohibée du sérialisme en plein fascisme, assumant le recours à cette technique comme une libération artistique mais aussi politique. D'autant que la genèse de l'opéra s'était élaborée dans le contexte d'un durcissement du pouvoir et d'une méfiance croissante du régime envers les avant-gardes ${ }^{1}$, et que l'épouse du compositeur Laura Dallapiccola de confession juive, était directement menacée par les lois raciales de $1937^{2}$.

Le Prisonnier est assurément une œuvre politique, au point de devenir aprèsguerre l'emblème des ouvres engagées. Mais sa dimension religieuse est tout aussi importante, car l'affrontement entre l'incarcéré et l'inquisiteur se situe sur le terrain de la foi, qui est l'occasion pour le compositeur d'affirmer une vision religieuse éminemment personnelle.

Que l'opéra se fasse l'écho d'une interrogation religieuse, là ne réside pas sa spécificité : la mise en musique de textes liturgiques témoigne d'une préoccupation constante du musicien, étalée sur l'ensemble de sa carrière et pas simplement limitée à la seconde guerre mondiale: Due laudi, 1929; Tre laudi, 1936-37; Requiescant (qui cite l'Evangile selon Saint-Matthieu) 1962, sans compter la Prière de Marie Stuart dans les Chants de Prison (1938-41), etc. Dans les questionnements communs à Job (1950) et Ulysse (1968), se révèle le refus

1 Durcissement esthétique symbolisé par la publication du Manifeste de 1932 signé entre autres par Pizetti, Respighi et Zandonai.

2 Le couple bascule dans la clandestinité à partir de 1940. C'est dans ce contexte qu'est composé l’opéra achevé en 1944 et créé en 1948. 
d'une stricte distinction entre textes religieux et profanes, voire catholiques ou païens.

Mais Le Prisonnier occupe dans ce corpus une place à part: l'Inquisition espagnole comme métaphore du pouvoir fasciste révèle une foi éminemment problématique lorsqu'elle devient le prétexte de l'écrasement de l'individu au nom de la religion, de sa manipulation pour l'amener à renoncer à ses dernières croyances, contre toutes les valeurs humanistes dont le compositeur avait déjà pu se faire l'écho dans ses Liriche Greche.

L'objet de cet article sera d'étudier la façon dont Luigi Dallapiccola décline les thématiques religieuses mais pour les retourner totalement, amenant même pour le croyant la possibilité de cesser de croire.

\section{L'INQUISITION, INSTRUMENT DE TERREUR ET MÉTAPHORE DU POUVOIR FASCISTE}

La première incarnation du religieux dans l'œuvre est l'Inquisition espagnole. L’analyse des intermèdes choraux qui lui sont consacrés fait émerger sa mise en lien avec le pouvoir fasciste. Comment parvenir à donner une identité musicale à une institution, dont le caractère abstrait pose problème sur une scène d'opéra? Tel nous semble être le rôle des intermèdes choraux: les personnages du geôlier et des deux moines sont des symboles de l'Inquisition mais celle-ci ne se réduit pas à eux. Elle possède une identité musicale, qui apparaît de façon frappante dans ces deux intermèdes. Langue, écriture chorale, puissance et hauts parleurs sont autant d'éléments qui la caractérisent. Alors que certains de ces éléments dénotent l'Inquisition du XVI ${ }^{\mathrm{e}}$ siècle, d'autres font signe clairement vers le pouvoir moderne.

Premier élément: la langue. Le chœur chante en latin, langue de la bonne doxologie, langue hors temps, hors de l'action, totalement étrangère aux personnages, dont le discours reste en italien, quelle que soit leur position vis-àvis du pouvoir ${ }^{3}$. Second élément: le chœur. Lors de la première intervention chorale, le contraste entre le monologue de départ et l'autorité du chœur est frappant : à l'enjeu personnel et la récurrence du «je» dans le discours de la mère: "Je le vois! Je le reconnais (...) je pousse un cri ...", répond l'universalité de l'église: "Que ta miséricorde s'étende sur nous». Et le texte choisi par Dallapiccola place d'emblée la religion comme garant de l'ordre («Que tes prêtres appliquent la justice»). Sur un plan musical, le discours du chœur s'oppose directement à celui de la mère: son figlio tenu sur un si bémol est doublement combattu par le la bécarre et par le si bécarre du chœur.

$\mathrm{Du}$ point de vue du langage, l'entrée de l'orgue, instrument hautement symbolique de l'église, soutient les deux sections, l'une homophonique, la seconde contrapuntique procédant par entrées d'un même motif à intervalles de demiton. Le discours recto tono, homosyllabique des premières mesures donne un caractère massif à l'ensemble, que la mise en musique du texte accentue: ainsi l'idée d'exultation («Et sancti tui exultent») est incarnée par une ligne recto tono

\footnotetext{
3 À l'instar du geôlier qui, devenu grand-inquisiteur, continue de parler italien.
} 


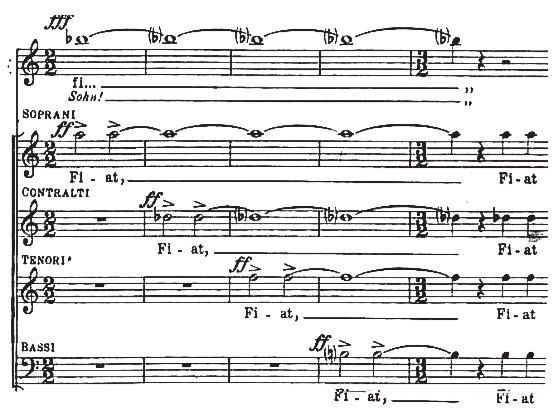

puis descendante qui entre en parfait conflit avec le sens du texte. Lorsque trois voix prononcent le terme "exultent», les cordes descendent d'une quarte diminuée. L'orchestration (trois timbales et grosse caisse mesure 139, suivie mesure 140 d'une descente chromatique aux contrebasses et violoncelles, registres uniformément graves pour tous les instruments) participe à une tension du discours, donnant au chœur un caractère véritablement effrayant.

Le second intermède choral reprend ces éléments tout en associant cette fois à l'institution ecclésiastique le régime fasciste par le recours aux hautparleurs et l'accentuation de la puissance chorale. La dimension collective se trouve là aussi opposée à l'individualité, grâce à l'écriture homophonique, et l'orgue réapparaît, associé à des chaines de tritons. Mais l'indication laissée par le compositeur dans la partition éclaire le sens de ces deux intermèdes: «la sonorité du deuxième intermède choral devra être formidable. Chaque spectateur devra se sentir littéralement emporté et submergé par l'énorme puissance du son. Il ne faut pas hésiter à se servir, si nécessaire, de moyens techniques tels que les haut-parleurs. " L'énorme puissance sonore due au climax orchestral qui évite lui aussi les registres aigus tout en ajoutant au premier intermède des «trombones sous la scène avec sourdine», ouvre pour l'auditeur le champ scénique et orchestral, lui donnant l'impression de "passer» dans un espace différent, celui du pouvoir.

Textes en latin, orgue, contrepoint, intervalles de tritons sont autant d'éléments qui associent symboliquement ces chœurs à l'église. La mise en lien avec le totalitarisme est, elle, nettement plus concrète et passe par l'emploi des hautparleurs: Dallapiccola "submergeant» l'auditeur en appelle à l'usage récurrent qu'en fait le fascisme. La prière en latin, scandée par un énorme chœur et amplifiée par des haut-parleurs évoque nombre de cérémonies populaires de la décennie 1930. Et la mise en lien des deux époques induit à l'évidence une dénonciation politique: le premier intermède rendait compte d'un climat de terreur propre à l'Inquisition espagnole ; après plus d'un siècle de dénonciation littéraire ceci n'a rien de particulièrement novateur. Le second intermède introduit lui, un décalage entre l'époque apparente et celle sous-entendue grâce aux moyens techniques, incongrus dans le cadre d'un discours centré sur l'Inquisition espagnole, décalage dont se nourrit la mise en cause du pouvoir. 


\section{LE RENVERSEMENT DES VALEURS CHRÉTIENNES}

«Selon moi, il faut conquérir la foi; or plus la conquête est difficile, plus elle a de valeur. 4 "

\section{Le retournement des vertus théologales}

Le Prisonnier ou le retournement diabolisé des valeurs chrétiennes ... Charité, espérance, parcours christique, les principaux motifs religieux sont au centre de l'opéra, mais de façon pervertie. Car l'Inquisition en utilisant successivement la compassion, la foi et l'espérance pour manipuler son fidèle, apparaît comme celle qui fait régner l'enfer sur terre.

Première des vertus théologales à être évoquée dans le drame, la charité, soit l'amour et la compassion, est aussi celle qui le fait basculer (en amenant le prisonnier à la fuite). Le motif mélodique particulièrement important qui lui est associé (fratello) capte l'attention dès son apparition grâce à son orchestration qui rompt avec le texte antérieur (ligne mélodique aux intervalles resserrés - moins d'une quarte - accompagnée de cordes sur des hauteurs gelées). L'évocation des mots du geôlier, précédée d'un silence, possède d'emblée une couleur orchestrale particulière: cordes dans l'aigu, harpe et célesta. La seconde occurrence du terme fratello, amplifiée orchestralement par les trilles de l'orchestre réitère une même phrase mélodique, offrant un parallélisme entre les derniers mots des deux phrases du prisonnier: «vita» et «luce».

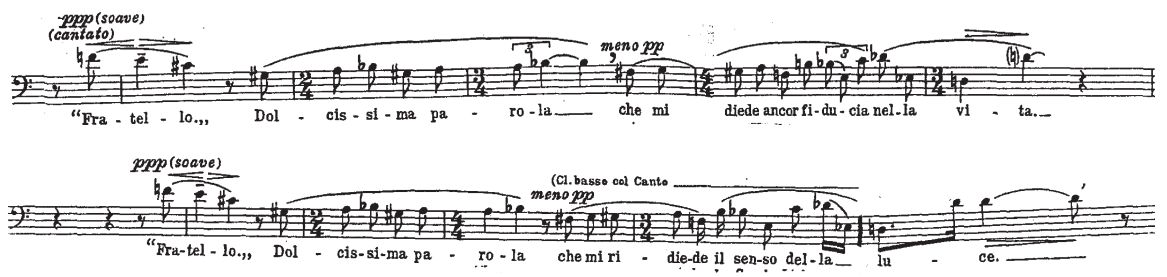

La charité permet le retour à la vie, elle contient aussi la possibilité d'un retour vers la foi. Il ne relève sans doute pas du hasard que "depuis ce soir», le prisonnier ait «recommencé à prier». Elle est au cœur du dénouement, en témoigne dans la partition la requête relative à la «douceur extrême» avec laquelle l'inquisiteur doit mener sa victime au bûcher.

La foi constitue le second motif récurrent, qu'elle soit incarnée par les prières collectives du chœur, la prière marginale du chœur final, ou bien celles du prisonnier lui-même. La première prière lors de son dialogue avec la mère directement issue des paroles du geôlier, est construite sur la série homonyme, soutenue par les accords de départ aux cordes, qui agissent comme un motif d'avertissement. La seconde prière intervient lors de la fuite, dans un contexte diatonique de $m i$ majeur associant à la série de la prière (original) celle de l'espoir, sur une ligne mélodique ascendante, a capella, imploration suivie d'un solo au violoncelle (le premier depuis le début de l'opéra). La troisième prière

4 Luigi Dallapiccola, entretien paru dans l'Espresso, 18 Juillet 1971. 
enfin, intervenant lors du dernier moment de désespoir du prisonnier, reprend le même motif mélodique d'imploration à Dieu, soutenu cette fois par tout l'orchestre, et contient une progression mélodique (arrivée sur un contre $f a$ pour le ténor). Mais l'orchestre énonce fortissimo les accords de début. À l'espérance du prisonnier répond le thème funèbre, qui combat sa prière au sens littéral du terme: mélodie individuelle contre homophonie, lyrisme contre total chromatique, collectivité contre individu. Cette foi est donc extrêmement individualisée, qu'elle soit chantée a capella, soutenue en sourdine par l'orchestre ou combattue par celui-ci, marquée enfin par une prégnance du diatonisme : l'une des rares allusions tonales intervient à la mesure 629, faisant contraste avec le chromatisme antérieur.

Dernière des vertus théologales, l'espérance est centrale. Elle est exactement ce vers quoi l'inquisition cherche à amener sa victime, pour mieux la briser. "Comment dire d'où nous vient l'espérance?» demande le fils à sa mère, et pour l'évoquer, d'utiliser une vocalité extrêmement lyrique, marquée par un agrandissement des intervalles (sixte, puis septième, avant une octave diminuée). "Comment s'insinue-t-elle dans nos cours?»: ascension mélodique soutenue par les contrebasses de deux intervalles de septièmes. Les premiers mots prononcés par le geôlier se rattachent à cette idée: «frère ... espère » et sont ensuite répétés de façon de plus en plus insistante: «Espère, frère, espère avec ardeur; tu dois espérer jusqu'à en souffrir, tu dois espérer à chaque heure du jour; tu dois vivre pour pouvoir espérer ». L'injonction du geôlier est incarnée par la rigueur de la construction musicale: imitations sur la série de l'espoir entre le violoncelle, le hautbois et le basson, tandis qu'un canon des cuivres énonce la série de la liberté. Ce canon est ensuite repris pour la répétition des ordres. Le caractère apparemment sacré de l'espérance est ici mis en valeur par le tissu contrapuntique, symboliquement accentué par l'organisation ternaire des «tu dois». La réponse du prisonnier après l'évocation de la révolte est du même registre: "Frère, merci à toi qui m’a redonné l'espoir !». Les derniers mots du geôlier parachèvent l'édifice: «dors ... et espère». C’est vers l'espérance que mène le parcours du prisonnier, afin de lui redonner la foi. Et c'est elle qui est anéantie à la fin de l’opéra.

Saisi par le Grand Inquisiteur, le prisonnier se trouve littéralement suffoqué. Après avoir compris l'enjeu véritable de sa manipulation, il est réduit à un ultime mot: «la liberté». Sa condamnation à mort est-elle symbole d'une libération? Dans une perspective strictement religieuse, certainement. Au niveau du drame et de ses implications symboliques rien n'est moins sûr: lorsque le geôlier évoque une libération par le salut du prisonnier ( frère ... à la veille de ton salut, tu voulais donc nous abandonner?»), celui-ci répète une première fois «avec inconscience» le mot de liberté, puis le prononce une seconde fois mais sur un ton interrogatif: «la liberta?». Affranchi d'une raison trompeuse, le prisonnier s'adresse désormais à une autorité divine: question sans réponse, où pointe un doute métaphysique. D'où la traduction musicale de cette interrogation, incarnée par une tierce descendante, ce qui est de l'ordre sinon de la contradiction, du moins du paradoxe. Comment interpréter une question descendante? La réponse est à trouver dans sa dimension symbolique: il n’y 
aura pas de libération dans la mort. La fuite du prisonnier l'a mené à un échec total, et la foi au nom de laquelle a été parcouru ce chemin de croix était une foi pervertie.

«Laissez ici toute espérance ... ${ }^{5}$ » L'Inquisition fait abandonner à l'individu l'espérance, la foi et pervertit la notion de charité. Ce retournement diabolique des valeurs religieuses manifeste une dénonciation virulente du chrétien visà-vis de l'église.

\section{Un parcours christique}

«Seigneur aide moi à marcher, le chemin est si long qu'il me semble que je ne pourrai arriver jusqu'au bout, Seigneur aide moi à m'élever». Mise à mort de son propre enfant par l'institution ecclésiastique, chemin de croix semé d'épreuves et de chutes, parcours menant de l'ombre des cachots à la lumière du bûcher, les métaphores portant sur le caractère christique des souffrances endurées par l'incarcéré sont récurrentes. À cet égard, le personnage universel de la mère possède un aspect éminemment chrétien, renforcé par le choix des textes cités; il est frappant que le compositeur ait cité une autre $\mathrm{Marie}^{6}$ : Marie Stuart. Telle une pietà, la mère, par son impuissance devant les souffrances de son fils, sa clairvoyance sur l'issue finale, accentue la dimension christique de l'opéra. Mettre en musique le sort d'un prisonnier-alter ego du Christ torturé par l'Eglise catholique témoigne de la défiance profonde du compositeur chrétien vis-à-vis de l'institution comme métaphore de tout pouvoir temporel. Selon Mila De Santis, «le lien étroit entre musique et religion tel qu'il s'élabore dans la poétique du compositeur, ne s'incarnera jamais dans la mise en musique d'un texte confessionnel.7 » Et pour le musicien lui-même, «Le projet de la Messe est, à mon avis, le plus difficile et le plus problématique qu'un musicien puisse se proposer d'aborder et, jusqu'à présent, je ne me suis pas senti assez mûr pour le faire ${ }^{8}$ ». Ainsi s'exprime Dallapiccola lors de son refus ${ }^{9}$ de composer une messe pour la cathédrale de Coventry (1964). La notion d'institution religieuse semble ainsi catalyser la méfiance du compositeur, qui lui substitue une autre foi individuelle, empreinte de doutes et de questionnements. Cette religiosité se heurte pourtant en 1948 à un constat d'échec absolu, car l'église du Prisonnier est aussi symbole de la destruction du message chrétien.

\section{COMMENT CROIRE?}

Le Prisonnier est l'histoire d'une manipulation ... Liberté intellectuelle et manipulation de l'autre sont au cœur de la problématique éthique de l'opéra. L’opéra peut être lu comme une éclatante démonstration du pouvoir de la parole: la simple occurrence du mot fratello et de son incarnation musicale suffisant

5 Dante 1985.

6 Le Prisonnier cite le premier des Chants de Prison : Prière de Marie Stuart

7 de Santis 2005.

8 Luigi Dallapiccola, Preghiere per baritono e orchestra da camera, in Parole e Musica.

9 Il diffère d'abord la commande pour terminer Ulysse, mais ne la réalisera jamais. 
à guider les actions et pensées des personnages. Transparaît également une puissante réflexion sur les mécanismes de renoncement au libre-arbitre.

\section{Destruction du libre-arbitre}

Le prisonnier est un corps souffrant: «ténèbres dans cette cellule, ténèbres dans mon cœur. Non, je ne savais pas que l'on pût tant souffrir sans en mourir.». Malgré l'enfermement et la confusion mentale, il est pourtant resté libre. L'intervention du geôlier consiste à le désorienter totalement, la pire torture n'étant pas celle qui détruit le corps, mais celle qui fait plier l'âme. Tout comme les inquisitions ibériques s'étaient donné pour but de sauver les âmes des prisonniers et d'y employer tous les moyens nécessaires, l'ultime quête du Grand Inquisiteur sera de briser moralement sa victime.

D'où l'importance du motif littéraire du regard, aussi important que le motif musical mélodique associé au mot "fratello ${ }^{10^{0}}$. L'âme au cœur des rapports de domination explique la référence récurrente au regard: qui voit clair? Que voit-on, que croie-t-on voir? Lors de son entrevue avec sa mère, le prisonnier parle des "ténèbres», des "ombres». Seul le mot «frère» lui redonna «le sens de la lumière». Le motif du regard semble dès lors résumer la lucidité (pris dans son sens étymologique) des personnages. Lors de l'évocation de la révolte de Gand, les couleurs sont également convoquées: «cygnes blancs qui virevoltent (...) trois couleurs pour l'étendard (...) le blanc pour la liberté, le bleu pour la gloire, l'orange pour le prince». En grossissant le trait, on pourrait même dire que c'est l'aspiration à re-voir qui pousse le prisonnier à sortir de sa cellule, aspiration encouragée par le mirage tendu auparavant. Le motif des yeux intervient à une seconde reprise, lors du seul instant de lucidité du fuyard (troisième ricercare) qui se demande si «les yeux terribles» l'ont vu. En soubassement de cette idée, peut-être y a-t-il le regard de Dieu auquel rien n'échappe. Ces yeux terribles, sont-ils ceux de l'inquisiteur ou bien ceux d'un Dieu plus terrible encore? À cela Dallapiccola n'offre pas de réponse précise. Les derniers instants du prisonnier restent marqués par cette emprise du regard, mais cette fois, «la lumière s'est faite». Non seulement le prisonnier voit vraiment, comprenant l'ampleur de la duperie, mais la lumière qui se fait est aussi celle du bûcher. Lumière mentale, lueurs de la mort imminente, impossible d'échapper à ce que l'on n'a pas voulu voir ...

En pensant voir clair à nouveau, le prisonnier s'est perdu lui, qui lorsqu'il ne voyait pas ("j'étais seul, tout était ténèbres»), pouvait encore juger. Le regard comme guide trompeur de la raison? Peut-être, mais la duperie est aussi et avant tout musicale. Cette non-lucidité amène donc à la question de la liberté, définissant en creux les caractéristiques d'un être libre: capacité d'autrui à le considérer comme individu, possibilité pour lui de choisir librement, liberté physique, enfin. C'est en tout cas ce que nous dit Dallapiccola, montrant que la manipulation mise en place par le pouvoir cherche moins à faire miroiter une libération factice qu'à tendre au prisonnier ces trois mirages les uns après les autres pour mieux en montrer la vacuité lors du dénouement.

10 Nous y reviendrons par la suite. 
Première illusion, la compassion. Abordée dès le dialogue entre le prisonnier et sa mère, elle est symbolisée par un unique motif: fratello qui, dès son énonciation (mesure 198) est associé à la série de l'espoir, jusqu'alors absente de l'opéra (mesure 201). L'espoir n'était pas possible avant le regard du geôlier. Le motif fratello, vocalement très caractérisé par son registre aigu, interrompt le dialogue entre la mère et son enfant. Celui-ci ne l'écoute plus, se bornant à répéter par trois fois les paroles du geôlier. Et lorsque la mère annonce la mort prochaine de son fils ("est-ce là, dis-moi, vraiment, notre dernier adieu?» mesure 265), il ne répond pas, trop occupé à entendre le motif énoncé au xylophone (mesure 270). L'unique instant de lucidité du prisonnier intervient lors de sa fuite, mais ne résiste pas au motif qui le tente à nouveau : «je n'en puis plus. (...) que faire? Retourner dans ma cellule obscure pour attendre encore, et toujours en vain?». Ce moment de doute est suivi de la première parole lucide de la part du fuyard: «j'entends une voix semblable autour de moi: de ces ténèbres elle m'appelle vers la lumière ... elle m'envoûte et m'attire à elle avec des mots magiques». Fratello ... mot magique qui élève l'âme du prisonnier et qui, comme il le formule si justement, l'envoûte. Sur le mot luce, l'intervalle mélodique est celui d'une tierce descendante ( $m i$ bémol-do, mesure 699), en parfaite contradiction avec le propos du texte, et constitue une troublante anticipation de l'intervalle final du condamné (la liberta? mi bécarre-do). Mais cet instant d'intelligence est immédiatement suivi dans l'intrigue, d'une reprise en main de l'institution qui vient contrecarrer la réflexion par son exact opposé: la terreur. Tel est le sens de la confrontation directe avec les deux moines, destinée avant tout à faire perdre le sens commun au fuyard.

Seconde illusion, la capacité d'agir librement, incarnée par la décision de fuir est, elle aussi "musicalement orientée » : après la visite du geôlier et l'appel à la révolte de la Chanson des Gueux, le prisonnier reste seul et aperçoit le reflet de la porte entrebâillée. Mais sa réaction a déjà été préparée, anticipée par l'agencement des motifs et des séries: le départ du geôlier est immédiatement suivi du rappel musical de la révolte des gueux à la clarinette (mesure 496), au sujet duquel le compositeur a demandé "come di lontano», comme de loin. Ainsi le monde extérieur se met-il à la portée de celui qui est enfermé. Dans le même temps, les cordes exposent le motif fratello. La réponse du prisonnier ne se fait pas attendre: l'espoir (mesure 501). Le reflet de la lampe, dont Dallapiccola précise qu'elle est celle du geôlier nous est rendu audible ... par le biais de ce motif auquel le personnage croit si aisément. Et lorsqu'arrive pour la première fois la série consacrée à la lumière, il semble naturel que l'hypothèse de la fuite prenne le pas sur la terreur.

C'est donc par un ensemble de "réseaux signifiants» associant des motifs à des sens, que Dallapiccola décrit le processus de manipulation psychologique auquel est soumis le héros. Et il est remarquable que ces «réseaux signifiants» se placent somme toute, sur un domaine exclusivement musical. Les séries n'accompagnent pas le discours. Elles l'enrichissent, l'anticipent, voire le contredisent, et fournissent des éléments essentiels d'explication à la psychologie du prisonnier. 


\section{Une autre foi?}

Face à la manipulation, deux discours s'opposent à ceux de l'Inquisition: la prière de Marie Stuart, sœur symbolique de prisonnier et l'intervention de la mère, sombre définition de la liberté.

La citation des Chants de Prison, première œuvre de la trilogie du "protest music» (Chants de Prison 1938-1941, Le Prisonnier 1941-1947, Chants de Libération 1955) intervient au plus fort de l'intensité dramatique: le prisonnier vient de comprendre l'enjeu de la duperie et s'apprête à se laisser guider vers le bûcher. Cet instant de lucidité se produit brutalement, en une secousse de trois mesures: immense crescendo ( $p p p p$ à $f f)$, sur une hauteur fixe, renforcé par une accélération brutale du rythme et un climax orchestral. À l'instar d'CEdipe, le prisonnier a compris la manipulation en un seul instant. De même que la tragédie grecque montre cet instant de conscience tragique dans lequel le héros découvre et assume son destin, l'orchestre incarne le retournement condensé de l'issue finale. Aucune place pour la mélodie, sérielle ou non : la série est de l'ordre du narratif, tandis que ces trois mesures cherchent à fixer un instant. Dès lors, les quelques mots prononcés par le prisonnier se font sur le motif fratello, pris désormais dans son sens véritable.

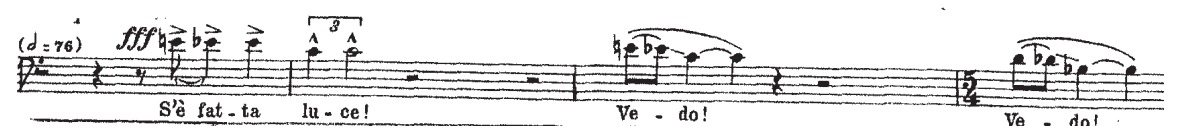

Le retour du grand chœur n'est que la reprise du second intermède choral, ultime commentaire de l'église avant la mise à mort. Dans un tel contexte, l'intervention des Chants de Prison sert d'intercesseur et de demande de compassion à un destinataire non précisé. D’où l'opposition complète entre les deux chœurs tant sur le plan de la mise en espace (demande expresse du compositeur que les deux soient placés face à face) que de la vocalité, de l'orchestration, et bien entendu du sens.

Sur un plan littéraire, l'opposition entre collectivité et individualité prend ici tout son sens: chœur de chambre, voix nues entrant en imitations pour la citation de Marie Stuart, grand chœur homorythmique en face. L'extrait choisi probablement pour sa force expressive par Dallapiccola contient trois occurrences du «je»: "dans ma faiblesse, gémissant, à genoux je me traîne», tandis que le chour reprend le texte du Psaume 50 issu de l'Ancien Testament, incarnation de la parole de Dieu: «et ma bouche continuera à chanter Tes louanges. ». Outre l'opposition entre mélodie du chœur de chambre et statisme du chœur religieux, s'opposent de façon complètement allusive deux mondes tonaux: les Chants de prison sont construits sur la superposition d'un langage sériel et du Dies Irae, mélodie diatonique par excellence. Cette juxtaposition confère au texte une charge symbolique évidente, née de la confrontation entre une mélodie extrêmement connotée, issue de la tradition, tournant sur ellemême d'une part, et la série ascensionnelle d'autre part, tentative d'élévation du chrétien. L'allusion au Dies Irae n'intervient pas dans le Prisonnier mais la 
citation des Chants en appelle imaginairement au conflit entre ces deux espaces harmoniques ${ }^{11}$.

Dernier sens possible au choix de ce poème plutôt que celui de Savonarole ou de Boèce (les deux autres textes choisis pour compléter les Chants): la reine d'Ecosse, emblématique symbole de l'emprisonnement devient en quelque sorte «la prisonnière», sœur du héros de Dallapiccola, appelant pour lui la miséricorde au moment où s'achèvent les souffrances. Mais cette ultime charité apparaît elle-même gangrenée de l'intérieur, grâce à la déconstruction de la série d'origine des Chants de Prison: au lieu de reproduire très exactement la construction de départ ${ }^{12}$, Dallapiccola ajoute une note qui modifie totalement l'équilibre mélodique initial: les deux intervalles de tierces mineures suivis d'une quinte deviennent désormais: tierce majeure, seconde majeure, seconde mineure, soit avec le demi-ton pour unité, 3-2-1, ce qui semble creuser la série de l'intérieur. S'impose alors une parenté avec la série des deux intermèdes choraux, dont le motif tiré de la série dite de la liberté13 possède le même incipit. Tirer une interprétation univoque d'un réseau si allusif forcerait le sens d'une telle musique. Il est cependant clair que la poétique dallapiccolienne se nourrit d'allusions, de renvois et de citations dont les modifications font sens, la nature exacte de celui-ci restant toutefois de l'ordre de l'indéfinissable. Dans le cas des Chants de Prison, la torsion imposée à la série de départ semble déconstruire la prière de la reine. Mais ceci n'est qu'une hypothèse ....

\section{Rester libre}

Le Prisonnier ou la démonstration du pouvoir de la parole ... Par la simple occurrence du mot fratello, l'inquisiteur parvient à brouiller la conscience du détenu et à le manipuler. Mais sa parole n'est pas libre pour autant: le marché de dupes proposé au nom de la foi la rend de fait viciée, corrompue de l'intérieur. La confrontation entre ces deux personnages montre, pour des raisons différentes, l'enfermement de leurs deux discours.

Seule la mère est un personnage libre dotée d'une parole vraie, car le geôlier pervertissant le message divin au nom du bien commun n'est pas plus libre que le prisonnier sacrifié pour avoir quêté sa liberté. À l'enfermement physique et mental de son fils, à l'enfermement dogmatique répond son monologue, seuls instants de l'opéra où les mots ne mentent pas. La parole de la mère est libre en ce qu'elle n'est ni manipulée ni manipulatrice et n'entre pas dans les rapports de domination geôlier-prisonnier. Le choix de lui consacrer le prologue la positionne en dehors de l'action, hors des scènes. Sa dimension christique est accentuée par la clairvoyance de son discours: la scène de rêve anticipe l'ensemble du drame à venir. Clairvoyance et non pas lucidité, car cette figure maternelle est

11 Avec des nuances tout de même : cette hypothèse reste de l'ordre de l'interprétation analytique, car le Dies Irae n'est pas cité dans l'opéra, l'allusion au conflit entre diatonisme et chromatisme relevant avant tout d'une construction symbolique.

12 ( $m i-s o l-s i$ bémol-fa)

13 Des titres ont été donnés aux quatre séries de l'œuvre ainsi qu'au motif musical récurrent «fratello », par le compositeur lui-même. 
non-seulement consciente des enjeux en cours, mais a anticipé la fin tragique qui attend son fils, et le rôle qu'y jouera l'incarnation de Philippe II.

Le récit de rêve incarne cet instant quasi-surnaturel dans lequel un personnage fait la lumière sur l'action à venir: alors qu'elle voit clair sur la nature de l'ombre en train d'émerger, son regard se trouve forcé: «quelque chose de plus fort que moi maintient mes pupilles ouvertes et fixes.». Mais cet aveuglement contraint n'entraine pas un aveuglement de la pensée, dans la mesure où Philippe II associé aux bûchers qui attendent son fils, change de visage et prend celui de la mort. Dès lors, la mère a compris que la proximité de l'Inquisiteur, incarnation du roi, conduira son fils au bûcher.

Le rôle important de la mère est souligné par un univers instrumental tout à fait spécifique : sa première intervention contraste avec la scène précédente sur le plan des textures orchestrales, autant que du dynamisme des scènes: univers nettement plus statique, chambriste, qui fait la part belle à la chanteuse, après la précipitation des accords du début. En un mot, le massif contre l'individuel. L'intensité de son discours est concentrée en six notes, les six premières, lancées sur quasiment deux octaves, et construites sur la série de la prière: incarnation d'une plainte désespérée, mais comme adressée au ciel. Et les trois réitérations du mot seront accompagnées d'une descente progressive: sur si (mesure 10) puis sur la (mesure 23) et sur sol (mesure 29), comme pour figurer de façon quelque peu littérale, une perte de confiance.
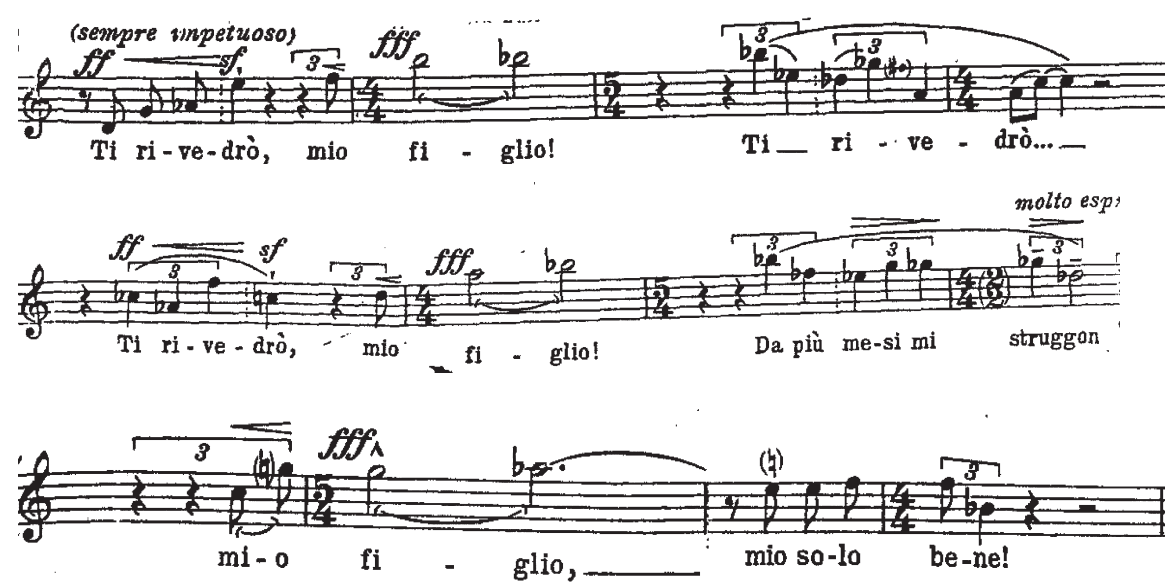

L'importance dramatique du rôle de la Mère est soulignée par une vocalité (toute puccinienne) et une orchestration spécifique qui lui associe la voix de la clarinette. Premier instrument entendu en soliste, la clarinette complète l'imploration par son motif ascendant. Lors de l'évocation du rêve, c'est elle qui donne à l'orchestration son aspect onirique (trilles en duo avec celles des cymbales suspendues) et introduit le «motif du rêve», au caractère immobile, gelé ("non expressif»), grâce à la réitération d'intervalles de tierces et de quartes au moment même où le compositeur réclame de l'interprétation qu'elle soit «visionnaire». Ce motif revient sur le mot «magie», mesure 106, aux clarinettes 
encore. Pourquoi une telle association d'un personnage à un timbre particulier? La Mère est, on l'a dit, un personnage à part. Sa vision, outre qu'elle permet la mise en abîme de l'opéra, lui confère un caractère surnaturel, accentué

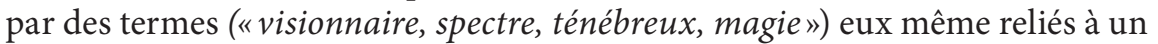
instrument. La série récurrente de ce prologue est celle de la prière, celles liées à l'espoir ou à la liberté n'ayant bien entendu absolument pas cours dans un tel contexte, car la Mère n'a plus d'espérance. Lors de la confrontation avec son fils, elle reprend d'ailleurs chacune de ses phrases, mais sur un ton interrogatif: "qui te redonna le sens de la lumière? (....) qui te redonna foi en la vie?». Le seul vrai moment dans lequel le prisonnier aura ce même ton interrogatif, sera lors de sa mise à mort («la liberta?). Il est donc possible de rester libre. Mais à quel prix. La perte d'un fils, le renoncement à l'espérance et le refus d' "entrer» dans l'action.

\section{Conclusion}

Telles qu'elles sont abordées, la dénonciation politique et la souffrance religieuse laissent entrevoir un engagement personnel de Luigi Dallapiccola, en humaniste chrétien et en individu directement touché par les lois raciales. L'analyse du Prisonnier ne peut être univoque: comme manifeste de la souffrance du compositeur lui-même, la critique religieuse qui s'en dégage reste allusive, discrète.

L'étude du pouvoir inquisitorial comme image du pouvoir fasciste est confirmée par des éléments musicaux et dramatiques, par la mise en exergue de ce qu'Hannah Arendt nomme des "conditions où la conscience n'est plus d'aucun recours, où bien faire devient radicalement impossible ${ }^{14}$ ». À travers la dénonciation d'un pouvoir politique devenu instrument de terreur, s'esquisse une définition de la liberté qui outrepasse largement l'enfermement physique. Le personnage de la Mère montre que la liberté n'échappe pas seulement à celui qui est enfermé, et que l'emprisonnement est partagé par le geôlier aussi bien que par son prisonnier. Mais cette liberté se situe d'emblée sur un terrain religieux: si l'enjeu politique est au centre de l'opéra, la question de la foi vient le compléter, voire le supplanter. Elle fait du prisonnier un Christ contemporain et laisse apparaître les doutes du compositeur lui-même.

Une vision très nuancée émerge donc de l'étude du Prisonnier, érigé comme emblème de l'engagement civique des artistes italiens. Avant d'être une charge politique, cet opéra n'est-il pas œuvre du doute et du questionnement? Que la violence exercée par une institution sur des individus ait trouvé une résonance fondamentalement politique semble aller de soi. Mais le chemin emprunté par le prisonnier met en jeu sa foi et se nourrit d'un questionnement métaphysique. Le message délivré par Dallapiccola rappelle donc l'importance de penser par soi-même, jusqu'au doute fondamental, quitte à perdre en chemin nombre d'illusions, d'espérances et jusqu'à la foi elle-même. Tel est le paradoxe du Prisonnier dont l'aspect politique masque peut-être encore l'errance existentielle. 


\section{RÉFÉRENCES}

Le Prisonnier. 2003. Avant-scène Opéra, n²12.

Le Retour d'Ulysse dans sa patrie. 1994. Avant-scène Opéra, nº 159.

Avdomikov, Paul. 1979. Dostö̈evski et le problème du mal. Paris: Éditions Desclee de Brouwer.

Bennassar, Bartolomé. 1979. L'Inquisition espagnole. Paris : Pluriel, Hachette.

Dallapiccola, Luigi. 1980. Parole e Musica. Milano: Il Saggiatore.

1992. Paroles et Musique, traduit de l'italien par Pierre Michel. Paris: Minerve.

Dante, Alighieri.1985. La Divine comédie, l'Enfer, Chant III. Paris: Garnier-Flammarion.

De Santis, Mila. 1997. Dallapiccola, Letture e prospettive. Pisa : Riccordi-LIM.

-2005. "Les expressions de la prière dans la musique de Dallapiccola». Musiques vocales en Italie depuis 1945, sous la dir. de Pierre Michel. Lillebonne: Millénaire III.

Dostoïevsky, Fédor. 1994. Les Frères Karamazov, traduction Henri Mongault. Paris : Folio classique.

Feneyrou, Laurent. 2003. Musique et dramaturgie: esthétique de la représentation au vingtième siècle. Paris: Publications de la Sorbonne.

Feneyrou, Laurent (dir.). 2005. Résistances et utopies sonores. Paris: CDMC.

Gentile, Emilio. 2004. Qu'est-ce que le fascisme? Histoire et interprétation. Paris: Folio histoire, Gallimard,

Arendt, Hannah. (1951 1963) 2002. Les Origines du totalitarisme. Paris: Gallimard, coll. "Quarto».

Michel, Pierre. 1996. Luigi Dallapiccola. Genève: Éditions Contrechamps.

-1990. La Musique vocale de Luigi Dallapiccola, techniques et esthétique, Thèse de musicologie, Paris IV, Direction Serge Gut.

Nicolodi, Fiamma. 1984. Musica e Musicisti nel Ventennio fascista. Fiesole: Discanto Edizioni.

Nono, Luigi. 2007. Ecrits, traduit de l'italien et de l'allemand par Laurent Feneyrou. Genève : Éditions Contrechamps.

Pena, Francisco. 2002. Le Manuel des inquisiteurs. Paris: Albin Michel.

Pavone, Claudio. 2005. Une guerre civile, essai historique sur l'éthique de la résistance italienne. Paris: Seuil.

Stenzl, Jürg. 1990. Von Giacomo Puccini zu Luigi Nono, Italienisches Musik, 1922-1952, Faschismus-Resistenza-Republik. Buren : Frits Knuf.

Villiers de l'Isle-Adam. 1990. Nouveaux contes cruels. Paris : Folio Poche.

Wachtel, Nathan. 2005. La Foi du souvenir, labyrinthes marranes. Paris: La Librairie du XXIème siècle, Seuil. Seuil.

\section{RÉSUMÉ}

Composé pendant la seconde guerre mondiale, Le Prisonnier de Luigi Dallapiccola constitue un tournant pour la musique italienne: premier opéra sériel italien en un 
temps de réaction esthétique et charge contre le fascisme, il est un modèle pour des compositeurs engagés tels que Luigi Nono et Bruno Maderna. Par-delà sa charge politique, Le Prisonnier est également l'expression d'une crise religieuse et d'un doute existentiel. L'Inquisition, institution religieuse par excellence est aussi celle qui torture le croyant au nom de la foi. Le retournement diabolique fait par le Grand Inquisiteur des principales thématiques chrétiennes vise à détruire le libre arbitre de l'autre. La manipulation du prisonnier, Christ contemporain, démontre la vacuité, voire la dangerosité de l'espérance, au nom de laquelle a été abjuré le libre arbitre. Dans l'affrontement entre le geôlier et son prisonnier s'esquisse enfin une sombre définition de la liberté car la parole de celui qui enferme n'est pas plus libre que celle de celui qui est enfermé. La mère seule, unique personnage clairvoyant de l'opéra a pu conserver sa liberté, mais à quel prix: celui de ne pas entrer dans l'action. Le doute qui émerge du Prisonnier est aussi celui de Luigi Dallapiccola, en individu directement touché par la guerre et les lois raciales, et en compositeur humaniste. L'objet de ce texte sera d'étudier la façon dont le musicien décline les thématiques religieuses mais pour les retourner totalement. Car Le Prisonnier, immense œuvre politique, est aussi celle de l'errance et du questionnement religieux.

\begin{abstract}
Composed during the Second World War, Luigi Dallapiccola's Le Prisonnier (The Prisoner) symobolized a turning point for Italian music, as it was the first Italian opera written with the method of serialism that is both an aesthetic work and an anti-fascist statement, it is a model for politically involved composers such as Luigi Nono and Bruno Maderna. In addition to its political nature, Le Prisonnier is also an expression of a religious crisis and existential doubt. The Inquisition is a renowned religious institution, but it also tortures believers in the name of faith. The Grand Inquisitor of Christian thematic principles conducts a diabolical reversal that seeks to destroy the free will of others. The manipulation of the prisoner, a contemporary Christ, demonstrates vacuity and even the danger of hope, for which they renounced their free will. In the confrontation between the jailer and his prisoner, a somber definition of freedom finally emerges, as the jailer's words indicate that he is no freer than the man whom he holds captive. The lone mother, a unique, perceptive character in the opera, managed to retain her liberty, but at a price: she could not become part of the action. Luigi Dallapiccola also embodies the doubt that arises from Le Prisonnier, he is directly affected by the war and by race-related legislation, and he is a humanist composer. The goal of this text is to examine the way in which the musician develops religious themes in order to completely transform them. Le Prisonnier, a massive political work, also examines wandering and religious questioning.
\end{abstract}

